NBER WORKING PAPER SERIES

\title{
URGENT CARE CENTERS AND THE DEMAND FOR NON-EMERGENT EMERGENCY DEPARTMENT VISITS
}

\author{
Lindsay Allen \\ Janet R. Cummings \\ Jason Hockenberry \\ Working Paper 25428 \\ http://www.nber.org/papers/w25428 \\ NATIONAL BUREAU OF ECONOMIC RESEARCH \\ 1050 Massachusetts Avenue \\ Cambridge, MA 02138 \\ January 2019
}

We thank participants in seminars at Emory University, West Virginia University, and the 2016 Southeastern Health Economics Study Group in Richmond VA for helpful feedback. This work was funded by a grant (R36HS2484501) from the Agency for Healthcare Research and Quality. The views expressed herein are those of the authors and do not necessarily reflect the views of the National Bureau of Economic Research.

NBER working papers are circulated for discussion and comment purposes. They have not been peer-reviewed or been subject to the review by the NBER Board of Directors that accompanies official NBER publications.

(C) 2019 by Lindsay Allen, Janet R. Cummings, and Jason Hockenberry. All rights reserved. Short sections of text, not to exceed two paragraphs, may be quoted without explicit permission provided that full credit, including $\odot$ notice, is given to the source. 
Urgent Care Centers and the Demand for Non-Emergent Emergency Department Visits Lindsay Allen, Janet R. Cummings, and Jason Hockenberry

NBER Working Paper No. 25428

January 2019

JEL No. I11

\begin{abstract}
Urgent care centers (UCCs) are a cost-efficient substitute to the emergency department (ED) for non-emergent conditions, but no study has identified their impact on ED demand. We address this gap using a novel strategy that exploits daily UCC operating times in a differencing framework. After UCCs close each day, local non-emergent ED visits increase by 1.43 percent (over the adjusted mean rate of 70.58 percent) in areas with multiple UCCs. This effect occurs only among the privately insured population, the target customers of UCCs. Our results suggest that UCCs are successfully substituting for EDs in the treatment of non-emergent conditions.
\end{abstract}

\author{
Lindsay Allen \\ West Virginia University \\ lindsay.allen@hsc.wvu.edu \\ Janet R. Cummings \\ Emory University \\ Department of Health Policy \\ and Management \\ 1518 Clifton Rd \\ Atlanta GA, 30322 \\ jrcummi@emory.edu
}

\author{
Jason Hockenberry \\ Department of Health Policy and Management \\ Rollins School of Public Health \\ Emory University \\ 1518 Clifton Rd \\ Atlanta, GA 30322 \\ and NBER \\ jason.hockenberry@emory.edu
}




\section{Introduction}

Reducing non-emergent emergency department (ED) use (i.e., visits that could have been safely delayed for 24 hours) is a well-known goal of health policy. Treatment for non-emergent conditions in the ED is more costly compared to that delivered in other care settings, without any concomitant rise in quality (Institute of Medicine 2007, Weinick, Burns, and Mehrotra 2010, Uscher-Pines et al. 2013a). Research indicates that up to half of the 137 million ED visits that take place annually in the U.S. could be treated at a care site other than the ED (Niska 2010, Young et al. 1996, Weinick, Burns, and Mehrotra 2010, Centers for Disease Control and Prevention 2011). A major barrier to treating these visits in a more clinically appropriate setting is limited access to acute care in the community, due to physician shortages, long wait times for appointments, and/or a lack of after-hours availability (O'Malley 2013, Kellermann 1994, Nadel 1993, Rask et al. 1994, Gindi, Cohen, and Kirzinger 2012, Cunningham 2006).

Urgent care centers offer a substitute care site for the treatment of unscheduled, acute, non-emergent conditions (Bohmer 2007, Mehrotra et al. 2009). Comprising an $\$ 18$ billion industry, these centers are freestanding facilities that provide after-hours and weekend care for injuries or illnesses that are "not life or limb threatening," but which are beyond the scope or availability of primary care facilities (Stoimenoff and Newman 2017, Urgent Care Association of America 2011). Care delivered in urgent care centers is less costly, more efficient, and of similar or better quality than that delivered in the ED (Weinick, Burns, and Mehrotra 2010, Institute of Medicine 2007, Uscher-Pines et al. 2013a, Bohmer 2007). Weinick et al. estimate that about 27 percent of all ED visits could take place at an urgent care center instead (Weinick, Burns, and Mehrotra 2010) .

Despite the potential impact of urgent care centers on healthcare cost and access, well identified estimates of their effect on ED demand are conspicuously lacking. Two empirical challenges account for this gap in the literature. First, there 
are data limitations concerning the timing of market entry and exit of urgent care centers, and unreliable measures of urgent care use. Second, a patient's decision to choose the ED versus an urgent care center is likely an endogenous one, partly determined by unobserved patient preferences or knowledge (e.g., about the ailments treatable at urgent care centers, whether their symptoms are lifethreatening).

Slightly more established is a mixed literature on retail clinics, for which data are more widely available and which share some similarities with urgent care centers, including walk-in availability and later hours of operation ${ }^{1}$. Alexander, Currie, and Schnell, use individual-level New Jersey data to examine the impact of retail clinics on ED visits for a small subset of six minor illnesses. They find that individuals living near an open retail clinic are between 4.7 and 11.4 percent less likely to go to the ER for these particular illnesses (Alexander, Currie, and Schnell 2017). Further, they estimate that expanding the retail clinic market across the state could result in an annual cost savings of over $\$ 70$ million from reduced ED use. Hollingsworth finds that Florida retail clinics decrease the number of ED visits for bronchitis and upper respiratory infection (Hollingsworth 2014). In contrast, Martsolf et al. find no impact of retail clinic penetration on low-acuity ED visits (Martsolf et al.). Given their small market size and the limited scope of services and resources they offer, retail clinics are not as close of a substitute for the ED as urgent care centers are. For example, urgent care centers offer diagnostic imaging and laceration repair, both of which are not provided in retail clinics (Stoimenoff and Newman 2017). This underscores the need for urgent care-specific research.

\footnotetext{
${ }^{1}$ Retail clinics differ from urgent care centers in that they are definitionally located within a retail location (rather than operating as a stand-alone entity), are much more limited in number, are often staffed by nurse practitioners (rather than MDs), treat a much smaller set of routine/minor conditions, have fewer clinical resources, and have lower out-of-pocket costs for patients.
} 
In this paper, we identify the effect of urgent care centers on demand for non-emergent ED visits. We employ a novel difference-in-differences strategy that uses daily opening and closure times of urgent care centers as proxies for market entry and exit. Hours of operation data were obtained from a dataset that has not previously been used in the literature. We merged this new, rich data source on urgent center operation times with ED visit records measuring the demand for nonemergent ED visits. Specifically, we compared pre- and post-daily opening/closure rates of non-emergent ED use in ZIP codes with urgent care centers to those in ZIP codes without urgent care centers, at the same time of day. Since urgent care centers are smaller than EDs and may require more than one clinic per area to meaningfully impact ED use, we divided our analysis into areas with single and multiple centers. We focused on treatment effects for patients who are privately insured, since they are the target market for urgent care facilities. In our framework, the uninsured population serves as a placebo test for our identification strategy. Unlike EDs, urgent care centers are not required by law to treat uninsured individuals, and therefore do not treat many individuals in this population (American College of Emergency Physicians 2018)

We estimate that the daily closure of nearby urgent care centers leads to a 1.43 percent increase in the rate of privately insured non-emergent visits immediately following closure. This extrapolates to about 2.4 million ED visits per year. Notably, this impact is only seen when there are multiple urgent care centers in a given geographical market. We do not find a meaningful effect of urgent care centers on the demand for non-emergent ED visits in areas with only one urgent care center, possibly due to capacity constraints of urgent care centers. As predicted, we find no effect of urgent care centers on non-emergent ED visits for uninsured individuals. Our results suggest that privately insured individuals living in communities with more than one urgent care center have lower non-emergent ED visits during the hours these centers are open. 


\section{Background}

\subsection{The Urgent Care Market}

Urgent care centers provide a potential ED substitute site of treatment of many acute, yet non-emergent conditions. In addition to primary care services, practitioners at these facilities can provide immunizations, lab tests, $\mathrm{x}$-rays, fracture and laceration care, and intravenous fluids (Urgent Care Association of America 2011). More than 7,600 urgent care clinics are in operation, with about 122 million annual patient visits (an average of 44 patients a day per facility), and hundreds of new clinics are opening each year (Urgent Care Association of America 2011, 2015, Stoimenoff and Newman 2017, Urgent Care Association of America 2017). In comparison, the 4,200 non-critical access hospitals (non-CAH) EDs in the US host 137 million visits per year ((an average of 89 patients a day per facility), and 1,800 retail clinics see only 10.5 million annual visits ((an average of 16 patients a day per facility) (Camargo and Sullivan 2018, Bachrach et al. 2015).

Despite its large size, the urgent care industry remains largely unregulated. To date, only Arizona and New Hampshire require urgent care centers to be licensed beyond what is required for any medical clinic (State of Arizona 2004, State of New Hampshire). Two states, Illinois and Delaware, regulate the use of the term "urgent care," but not the facilities themselves (Urgent Care Association of America 2015). Other than these exceptions, clinics can choose to label themselves as urgent care centers, or as any one of a number of related designations including "immediate care," "convenient care", and "walk-in care."

The Urgent Care Association of America (UCAOA) delineates urgent care centers from other delivery models based on whether a facility 1.) is open on weekday evenings and on weekends, 2.) does not require an appointment, 3.) has onsite x-ray, and 4.) has the ability to perform suturing and casting procedures (Urgent Care Association of America 2015). Nonetheless, services offered in an urgent care center can vary widely, from primary care to less common offerings, 
such as occupational medicine, weight loss, and physical therapy services (Urgent Care Association of America 2015).

This variability in regulation and designations makes it difficult collect valid data on the urgent care market, especially concerning when urgent care centers enter and/or exit geographic markets. Therefore, identifying their effect on the demand for ED visits is difficult using conventional yearly panel data approaches. Another data-related challenge is that insurance claims - often used in health care research - do not always identify which services were offered under the auspices of urgent care. Though there are some after-hours and other urgent carespecific insurance codes, which are pre-negotiated with an insurer, most urgent care billing relies on the evaluation and management codes also used in other primary care settings (Urgent Care Association of America 2015). Despite treating almost as many patients annually than all U.S. EDs combined, urgent care center status is also not captured in any large-scale national surveys, like the National Ambulatory Medical Care Survey (for primary care visits) and the National Hospital Ambulatory Medical Care Survey (for ED visits).

\subsection{The Role of Patient Preferences}

Adding to the problem of data scarcity, unobservable patient preferences make it difficult to establish the causal impact of urgent care centers on nonemergent ED use. Given the choice between receiving treatment at an urgent care center and an ED for a non-emergent acute condition, a patient may choose one over the other for reasons unknowable to a researcher in the limited data available. On the one hand, patients may prefer to visit an urgent care center due to perceptions that urgent care centers offer shorter wait times and lower-cost treatment. On the other hand, patients may instead choose to visit the ED, perhaps because they are open around the clock, easily recognizable and accessible, or perceived as offering more sophisticated or better quality treatment. 
An additional key dimension of patient preferences has to do with one's ability and/or willingness to act as a first-line diagnostician of their own condition. Before deciding to go to an urgent care center instead of an ED, a patient needs to have some idea of the seriousness of their condition, along with an idea of what clinical resources will be needed to treat it. Some patients may be unable or unwilling to conduct this initial triage process - thereby defaulting to the ED while others may have more experience or knowledge that allows them to select their optimal care site (Uscher-Pines et al. 2013b, Jaffe, Kocher, and Ghaferi 2018).

\subsection{Empirical Framework}

We address these challenges by adopting a novel difference-in-differences strategy that hinges on daily closure times of urgent care centers. In markets with an urgent care center, demand for acute, non-emergent care is spread across a larger care supply of facilities (i.e., the urgent care center and the ED), as long as the urgent care center is open. Once this center closes, the latent demand for acute services is spread over a smaller number of suppliers. We would expect this to manifest as an increase in non-emergent ED visit rate immediately after urgent care closure.

Conversely, we would expect a decrease in the non-emergent ED visit rate when urgent care centers open in the morning. However, the advantage of urgent care centers is that they are open on weeknights and weekends, when other care sites are not. In the morning, there are many different options for patients seeking non-emergent care (such as primary care physicians' offices that offer same day appointments, or school clinics). Therefore, we do not anticipate that urgent care centers will have the same impact in the morning that they would in the evening, when they close later than these other options do, becoming the only alternative to the ED.

Our empirical approach obviates the need for year-to-year panel data on urgent care centers, and instead addresses the endogeneity from unobserved patient 
preferences that affect observed visits using hours of operation data. For ZIP codes with an urgent care center, we compare rates of non-emergent ED use immediately before and immediately after those urgent care centers open for the morning, or close for the evening. If urgent care centers are effectively drawing non-emergent visits from EDs during the hours they are open, we would expect an increase in non-emergent ED visits when those facilities close for the evening. We would not expect a similar jump during the same time period in patient ZIP codes without an urgent care center, which comprise our control group. Taking this reduced form approach to estimate the effect on ED visit demand eliminates the need for data on urgent care visits, which are hard to come by, as noted previously.

Since urgent care centers are smaller than EDs, it is unlikely that a single urgent care center will drive meaningful change in nearby ED use (Stoimenoff and Newman 2017, Welch et al. 2012). For this reason, we divide our sample into two parts: those with exactly one urgent care center, and those with more than one urgent care center. For the analysis with multiple urgent care centers, we use the latest opening/closure time of all the clinics in the ZIP code as our cutoff point.

Unlike EDs, urgent care centers are not bound under the Emergency Medical Treatment and Labor Act (EMTALA) to treat all patients, regardless of their ability to pay (American College of Emergency Physicians 2018). This means that profit-maximizing urgent care centers can preferentially treat only those patients who can afford to pay for care, either through their insurance plan or outof-pocket. As such, urgent care centers and EDs have very different payer mixes. Whereas $67 \%$ of urgent care centers visits are by the privately insured, only $12 \%$ of visits are by uninsured individuals who are able to self-pay at the time of the visit (Urgent Care Association of America 2017) . In the ED, these percentages were $33 \%$, and $20 \%$ at the time our data were collected (Ashman, Rui, and Hing 2016). Given these numbers, we would expect urgent care center closures to have a measurable effect for patients who are insured, but not for patients who are 
uninsured. For this reason, we limit our main difference-in-differences analysis to privately insured individuals, and use the uninsured population to conduct a placebo test of our model. We exclude publicly-insured individuals $(21 \%$ of urgent care visits) from our models, since they are not the target demographic of urgent care centers, but represent a large enough patient base to invalidate their use as a placebo test (Urgent Care Association of America 2017).

\section{Methods}

Our data on urgent care centers came from a database, previously unused in the literature, which contains the names, addresses, and hours of operations for almost all of the urgent care centers in the U.S. The dataset comes from an online searchable database that allows patients to view the urgent care centers within a certain distance of their ZIP code. The dataset represents the most comprehensive, independently-verified directory of walk-in clinics in the country, with over $90 \%$ of clinics indexed (Urgent Care Location 2013, Barber 2015). The 2013 edition of the database, which we used for this study, contains 6,655 urgent care centers, which is in line with numbers released from industry trade organization estimates at that time (Urgent Care Association of America 2014). Clinic addresses and hours are regularly updated via a call center (Barber 2015). Because our identification strategy hinges on hours of operation, we removed from our list of facilities any clinics that did not have hours available in the dataset (17\% of the list).

Emergency department visit data come from the Healthcare Cost and Utilization Project (HCUP) State Emergency Department Databases (SEDD), which is the largest collection of all-payer, encounter-level ED visit data in the U.S. (Steiner, Elixhauser, and Schnaier 2001). For states choosing to participate in the HCUP, the data contain information on every ED visit that did not result in a hospital admission (Steiner, Elixhauser, and Schnaier 2001). The data include patient and visit characteristics, such as patient ZIP code, payer status (i.e., Medicaid, Medicare, private, uninsured), discharge code (ICD-9), and hour (but not 
minutes) of ED visit (arrival time, not admission time). Six states (Arizona, Florida, Nebraska, New Jersey, New York, and Rhode Island) included all of the variables necessary to implement our analysis. We used data from the year 2012, the most recent year for which all required variables were available. We aggregated the encounter-level ED records to the ZIP code level for our analysis.

To determine which visits are non-emergent, we used a recently updated version of the NYU ED visit algorithm, which classifies the urgency, preventability, and optimal care site of ED visits (Billings, Parikh, and Mijanovich 2000, Feldman 2010, Johnston et al. Forthcoming). For all discharge (ICD-9) codes available in the SEDD, the algorithm assigns a probability that the visit fell into one of four categories: 1) not an emergency; 2) emergent, primary care treatable; 3) emergent, ED care needed, but preventable/avoidable; 4) emergent, ED care needed, not preventable/avoidable (Billings, Parikh, and Mijanovich 2000). The probability can be distributed across the four categories, reflecting the variability of possible urgency levels within any single code ${ }^{2}$. We sum across the first two categories (not an emergency; emergency, primary care treatable) to obtain the probability that any given visit is non-emergent and could have been treated in an urgent care center.

When the algorithm was created, visits due to injury were assigned a probability of $100 \%$ to a carved-out injury category (instead of a one of the four urgency categories described above) $)^{3}$. Therefore, we do not have information on whether injury-related visits were non-emergent. For this reason, our main analytic sample excludes injury visits. However, the urgent care model is designed to treat

\footnotetext{
${ }^{2}$ For example, an ICD-9 code of 0340 , (streptococcal sore throat) has the following probability distribution: non-emergent, $66 \%$; emergent, primary care treatable, $28 \%$, emergent, ED care needed, $6 \%$; emergent, ED care needed, not preventable/avoidable, $0 \%$.

${ }^{3}$ Visits related to drug/alcohol use, and psychiatric related visits, were also carved out into their own categories. These carve-outs were conducted at the request of early ED algorithm users, so that they may be tabulated separately.
} 
minor injuries (sprains, minor fractures, etc.), and removing them completely from the analysis may be unrealistically restrictive. Therefore, we run sensitivity analyses in which we include injury visits, assuming varying percentages $(25 \%$, $50 \%, 75 \%, 100 \%)$ are non-emergent.

The SEDD does not provide the day of the week on which a visit occurred; it only provides whether the visit occurred on a weekday or weekend. Urgent care center hours tend to be very consistent from Monday through Friday, but change markedly from the work week to Saturday, and then again from Saturday to Sunday. For this reason, we excluded weekend visits $(28.1 \%$ of the records) from our analysis. To further derive our ED sample, we dropped those visits for which there was no visit time data ( $1.1 \%$ of the sample) and no payer information $(0.1 \%)$. Finally, we dropped all visits that occurred by people with missing or invalid ZIP codes (0.8\%), out of state home ZIP codes (3.4\%), and records that had ZIPs with only 3 digits $(0.2 \%)$. After dropping observations ( $4.5 \%)$ carved out by the NYU algorithm (i.e., those due to drugs, alcohol, or psychiatric conditions) and limiting the sample to privately insured (uninsured) adults age 0-64, we were left with 3.4 (2.4) million individual ED records.

We collapsed the individual-level SEDD data to the ZIP code level. Each ZIP code is represented multiple times in our dataset, since we observe the ZIPlevel non-emergent visit rate at each hourly time point.

We used data from the 2013 American Community Survey (ACS) to obtain descriptive statistics for ZIP codes with and without urgent care centers (United States Census Bureau 2013). Because ACS data are provided at the ZIP code tabulation area (ZCTA) level, we cross-walked the data values to their corresponding ZIP codes using a relationship file from the Missouri Census Data Center (Missouri Census Data Center 2016).

We first plotted the unadjusted zip-code level rates of non-emergent ED visits by hour, in ZIP codes with and without urgent care centers. This confirmed 
our identifying assumption that ZIP codes with and without urgent care centers have similar pre-trends in non-emergent ED visit rates prior to the daily opening/closure time.

More formally, we estimated a difference-in-differences model in which the change in the non-emergent visit rate before and after daily urgent care opening/closure time in ZIP codes with an urgent care center is contrasted with that same change in ZIP codes without an urgent care center. We examined the change in non-emergent ED rate in the hour just before versus the hour just after the opening/closure time threshold. Our model is

$$
Y_{i h}=\alpha+\beta_{0} T_{i}+\beta_{1} C_{i}+\beta_{2}\left(T_{i} \cdot C_{i}\right)+\varepsilon_{i}
$$

where $Y_{i}$ is the rate of non-emergent ED visits for ZIP code $i$ at hour $h ; T_{i}$ is an indicator equal to one if the ZIP code was assigned to the treatment group (i.e., has an urgent care center); $C_{i}$ is an indicator equal to one if the visit occurred at the urgent care closure time or later (or the opening time or later); $\left(T_{i} \cdot C_{i}\right)$ is the interaction between the two, allowing for different slopes on either side of the closure (opening) time cut point, $X_{i}$ is a vector of ZIP code characteristics; and $\varepsilon_{i}$ represents a random error term. Our key policy parameter is the coefficient on the interaction, which estimates the causal impact on non-emergent visit rates attributable to urgent care centers closing for the day in ZIP codes with an urgent care center.

Because there are likely unobserved factors that could be simultaneously impacting our independent and dependent variables, we include ZIP code-level fixed effects to capture any time-invariant, ZIP-code specific characteristics that might bias our estimate of the effect of UCC availability on ED demand. All models included dummy variables for each state. Standard errors were clustered at the Hospital Service Area (HSA) level, which can be thought of as roughly equivalent to a hospital's catchment area (Dartmouth Atlas of Health Care 2018). We weighted 
our estimate using analytic weights (command aweights in Stata) that we created according to the observed number of visits coming from each ZIP code.

To determine if ZIP codes with greater urgent care supply might present a larger impact on non-emergent ED use, we conducted sub-analyses in which we limited the sample first to those ZIP codes with exactly one urgent care center or no urgent care center, then to those with more than one urgent care center or no urgent care center.

The choice of opening and closure times for our analyses warrants discussion. Operating times for urgent care centers vary across locations, but we needed a single closure (opening) time to cleanly compare visit rates across all of our control ZIP codes. For example, if there were three ZIP codes, each with a UCC that closed at a different hour, we wouldn't be able to choose a single time point in our control group to make the comparison. For this reason, we focus our analysis on the modal closure (opening) time across all UCCs. The modal closure time for all UCCs in our sample across all weekdays is $8 \mathrm{PM}$, and the modal opening time is 8 AM. We first divided our "closure" ZIP code sample into those that had least one UCC $(n=835,15 \%)$ and those that did not have any UCCs $(n=4,754,85 \%)$. Within the group that had at least one UCC, we further divided the ZIP codes into those that had exactly one UCC $(544,65 \%)$ versus those that had more than one UCC (291, 35\%). Of the 544 ZIPs with exactly one UCC, 267 (49\%) closed at 8 PM. Of the ZIPS with multiple UCCS, 129 (44\%) had 8 PM as the latest closure time among the UCCs. ZIP codes with one UCC that did not close at $8 \mathrm{PM}$, or multiple UCCS that closed later than 8 PM, were excluded. We created the "opening" ZIP code sample similarly, using a "first opening" time of 8 AM in ZIPs where there were more than one UCC.

For each weekday, the modal UCC opening time (8:00 AM) accounted for over $54 \%$ of our sample of urgent care centers. The next most common opening times were 9:00 AM and 7:00 AM, accounting for 18\% and 6\% of the sample, 
respectively ${ }^{4}$. The modal weekday closure time (8:00 PM) represented over 39\% of the sample. The next most common closure times were 9:00 PM and 7:00 PM, representing about $14 \%$ and $11 \%$ of the sample, respectively. Openings and closures at times other than on the hour (e.g., 8:15, 8:30) were rare in the sample, but where they existed, we rounded that time down or up to the closest hour because the SEDD visit records only provide time of visit by the hour, not the minute. We conducted sensitivity analyses testing whether our results were robust to using the next most common opening and closure times (7

AM, 9 AM; 7 PM, 9 PM) as our time thresholds. We also tested for robustness of our main result to the size of the hourly window around the closure time threshold.

\section{Results}

Table 1 shows unadjusted descriptive statistics for ZIP codes with and without urgent care centers. Of the 5,589 ZIP codes in our sample of six states, $14.9 \%$ have at least one urgent care center. Areas with urgent care centers are much more populated than those without. They have a younger, more racially diverse, wealthier, and more educated populations. This likely reflects the tendency of walkin clinics to locate in more urban areas, relative to rural areas. ZIP codes with urgent care centers have lower rates of Medicaid enrollment, but higher rates of uninsured individuals, compared to those without urgent care centers.

In Figure 1, we present the mean hourly non-emergent ED visit rate for ZIP codes with and without urgent care centers. The dashed lines on the figure represent the modal weekday opening (8 AM) and closing (8 PM, or 2000 hours in military time) times among urgent care centers in our sample. In the hours leading up to these opening and closure times, the non-emergent visit rate rises and falls similarly

\footnotetext{
${ }^{4}$ We use Tuesday - Friday hours to get these hourly distributions, since hours remain virtually constant during these weekdays. Monday hours differ slightly. We use Tuesday hours for the analysis, since Tuesday hours have the fewest number of missing data points and are most similar to the rest of the weekdays' hours
} 
across areas with and without urgent care centers. This provides evidence that our data satisfy the parallel pre-trends assumption upon which our identification approach rests. Across both groups, we find that over 70 percent of ED visits are not true emergencies. Though estimates of non-emergent ED visits vary widely, these numbers are on the high end. This is likely because other studies only consider the least emergent (i.e., routine; those that could take place in a primary care office) visits in their estimates. Our study includes these visits, plus those that are too serious to be treated in a primary care office, but can be successfully treated in an urgent care office.

Table 2 presents results from our main difference-in-differences analysis. We first focus on the privately insured population. As shown in Panel A, we find no impact of urgent care centers on ED use when they open in the morning, even when there is more than one center in a ZIP code. This is consistent with our hypothesis that demand changes during this time frame will likely be spread across a greater provider supply, since other sources of non-emergent health care, such as primary care physicians' offices, tend to begin their workdays around the same time as urgent care centers. The hallmark of an urgent care center is its extended hours on weeknights and weekends - not its weekday morning hours.

In contrast, we find a statistically significant increase in non-emergent ED visits among privately insured individuals in ZIP codes with multiple urgent care centers, when the last center in that area closes for the day. Specifically, nonemergent ED visits rise by 1.01 percentage points $(p<0.01)$, a 1.43 percent relative increase. When viewed in context of the 136 million annual ED visits that take place in the US, this translates to an additional 1.9 million ED visits.

We do not find a statistically significant impact in areas with only one urgent care center, possibly because their capacity is too small to meaningfully impact visits rates in the ED. To provide a concrete example of this constraint, we performed a back of the envelope calculation. Urgent care centers treat about 44 
patients a day, while non-Critical Access Hospitals EDs are fielding an average of 89. Of those 89 patients, about 70\% (62 patients) could be treated in a UCC. Our results suggest that $1.43 \%$ of the 62 non-emergent ED patients (or just under 2 patients a day in a ZIP code with 2 UCCs) are being seen in the UCC, rather than the ED.

We used ED visits among the uninsured as a falsification test for our results. Because urgent care centers primarily cater to the privately insured, we would not expect their daily closure to impact ED use among uninsured individuals, who comprise only $12 \%$ of urgent care patients. This is confirmed in the last two columns of Table 2 . We find no statistically significant impact of urgent care center closure on ED use by uninsured patients.

We ran three sets of sensitivity analyses. One shortcoming of the algorithm we used to determine ED visit urgency is its exclusion of injury visits, an important consideration since urgent care centers are intended to treat a range of moderate injuries. When we included the injury visits in our sample, classifying varying percentages of them as non-emergent, we find a statistically significant effect at the $75 \%$ and $100 \%$ thresholds. When smaller portions (i.e., $25 \%, 50 \%$ ) of the injury visits are considered non-emergent, we find no effect (Table 3). To put these rates in perspective, the Agency for Healthcare Research and Quality finds that $90 \%$ of injury visits in the ED are "mild" (as opposed to moderate or severe), and almost all of these are treated and released (Villaveces et al. 2013). Taken together, this suggests that urgent care centers are particularly effective ED substitutes for patients with mild injuries.

We also attempted to test whether our main finding was sensitive to the daily closure/opening time chosen by running our models for the next most common opening and closure times (7 AM, 9 AM; 7 PM, 9 PM). Using these time thresholds, we did not find a statistically significant change in ED rates. We posit that this is because so few of the UCCs in our sample open and close at these less 
common times. For example, the 8 PM closure time accounts for over half the sample of urgent care centers, while the next two most popular closing times combined account for less than a quarter of the sample. If this is the case, it would support our theory that urgent care centers are most effective in reducing ED use when there are multiple centers available to patients.

Though we would expect to see the largest impact of UCC closure in the time window encompassing the hour immediately before and the hour after closure, we also tested the robustness of our finding to a wider time window. The results hold at both two hours before and after closure (i.e., a four-hour window; 0.80 relative increase, $\mathrm{p}<.10$ ), and three hours before and after closure (i.e., a six hour window; 0.74 relative increase, $\mathrm{p}<.10$ ), in areas with more than one UCC. The magnitudes of the effects, however, decrease as the time windows get larger, which is to be expected.

\section{Conclusions}

Urgent care centers offer a potentially important substitute care site for nonemergent ED use, but systematic evidence of their impact has been lacking due to data challenges and concerns about unobserved patient preferences. We addressed these challenges by implementing a difference-in-differences model that uses daily operation times of urgent care centers as proxies for market entry and exit.

In a multistate analysis that used a novel data source, we found that daily closure of urgent care centers meaningfully increases privately insured nonemergent ED use in hours that follow, as long as there are multiple urgent care centers in the area. We do not find corresponding impacts in areas with a single urgent care center, which we attribute to capacity constraints. As predicted, we do not find corresponding impacts for uninsured patients, a group comprising a very small part of the urgent care case mix. 
The effect size we find translates to approximately 2.4 million ED visits per year. Since it costs around $\$ 414$ more to treat a non-emergent case in an ED versus an urgent care center, this represents about $\$ 1$ billion in health care costs, annually.

While this is the first known study to assess the causal impact of UCCs on non-emergent ED visits, we can place our findings in the context of the emerging literature on retail clinics, a smaller and more limited market. Alexander, Currie, and Schnell find that individuals living close to an open retail clinic are up to 11.4 percent less likely to go to an ED for sprains and strains, urinary tract infections, conjunctivitis, upper respiratory tract infections, ear infections, and sore throat (Alexander, Currie, and Schnell 2017). Hollingsworth finds that Florida retail clinics decrease the number of ED visits for bronchitis and upper respiratory infection (Hollingsworth 2014). Taken together, these studies suggest that the walk-in clinic industry (comprising urgent care centers and retail clinics) has the potential to meaningfully reduce non-emergent ED visits, which in turn may reduce health care expenditures. Indeed, Sussman et al. find that individuals who used a retail clinic had lower care costs over the following year, compared to individuals who received care in other settings(Sussman et al. 2013). On the other hand, another suggested that retail clinics may offset these health care savings by encouraging visits along the extensive margin (i.e., those who would have forgone any care if a retail clinic were not open at the time of visit) (Ashwood et al. 2016). Additional research is needed to identify the net effect of the walk-in industry on health care costs.

Our results may represent an upper bound on the impact of clinics on ED use: first, the 7,600 clinics in operation today are probably located where they expected to find the greatest privately insured patient demand. As the market matures, there may be diminishing returns to opening new clinics. Secondly, the evening portion of our analysis takes place during a time of day where ED visits are high (Pitts et al. 2012). Because the number of visits is smaller at other times of 
the day, there are fewer visits for the walk-in clinics to impact. On the other hand, only about $5 \%$ percent of ZIP codes (in our sample) have more than one urgent care center. Since we find that meaningful change in ED use occurs only when multiple centers locate in one area, urgent care companies may be incentivized to increase their supply in certain areas, which would bolster their impact. 


\section{References}

Alexander, Diane, Janet Currie, and Molly Schnell. 2017. Check Up Before You Check Out: Retail Clinics and Emergency Room Use. National Bureau of Economic Research.

American College of Emergency Physicians. 2018. "EMTALA." https://www.acep.org/life-as-aphysician/ethics--legal/emtala/emtala-fact-sheet/\#sm.00001m2o2n7hovdd6t8x1 eqactduh.

Ashman, Jill J, Pinyao Rui, and Esther Hing. 2016. Expected Source of Payment at Emergency Department Visits for Adults Aged 18--64 for the United States and in the Five Most Populous States, 2012: US Department of Health \& Human Services, Centers for Disease Control and Prevention, National Center for Health Statistics.

Ashwood, J. S., M. Gaynor, C. M. Setodji, R. O. Reid, E. Weber, and A. Mehrotra. 2016. "Retail Clinic Visits For Low-Acuity Conditions Increase Utilization And Spending." Health Aff (Millwood) 35 (3):449-55. doi: 10.1377/hlthaff.2015.0995.

Bachrach, Deborah, Jonah Frohlich, Allison Garcimonde, and Keith Nevitt. 2015. "The value proposition of retail clinics." Robert Wood Johnson Foundation:12.

Barber, Michael; . 2015. "Personal Communication with Michael Barber." June 12, 2015.

Billings, J., N. Parikh, and T. Mijanovich. 2000. "Emergency department use in New York City: a survey of Bronx patients." Issue Brief (Commonw Fund) (435):1-5.

Bohmer, Richard. 2007. "The Rise of In-Store Clinics — Threat or Opportunity?" New England Journal of Medicine 356 (8):765-768. doi: doi:10.1056/NEJMp068289.

Camargo, Carlos;, and Ashley; Sullivan. 2018. National Emergency Department Inventory - USA.

Centers for Disease Control and Prevention. 2011. National Hospital Ambulatory Medical Care Survey: 2011 Emergency Department Summary Tables.

Cunningham, P. J. 2006. "What accounts for differences in the use of hospital emergency departments across U.S. communities?" Health Aff (Millwood) 25 (5):w324-36. doi: 10.1377/hlthaff.25.w324.

Dartmouth Atlas of Health Care. 2018. "Research Methods." accessed October. http://www.dartmouthatlas.org/downloads/methods/research_methods.pdf.

Feldman, J. . 2010. The NYU Classification System for ED Visits: WSHA Technical Concerns. Washington State Hospital Association: Health Information Program.

Gindi, Renee M, Robin A Cohen, and Whitney K Kirzinger. 2012. "Emergency room use among adults aged 18-64: early release of estimates from the National Health Interview Survey, January-June 2011." National Center for Health Statistics.

Hollingsworth, Alex 2014.

Institute of Medicine. 2007. Hospital-Based Emergency Care: At the Breaking Point: The National Academies Press.

Jaffe, Todd A., Keith E. Kocher, and Amir A. Ghaferi. 2018. "Potentially Avoidable Emergency Department Use: When Policy Expects Patients to be Physicians." Annals of Emergency Medicine 72 (3):256-258. doi: 10.1016/j.annemergmed.2018.05.019. 
Johnston, K., L. Allen, T. Melanson, and S. Pitts. Forthcoming. "An Update to the NYU Emergency Department Algorithm." Health Serv Res.

Kellermann, A. L. 1994. "Nonurgent emergency department visits: Meeting an unmet need." JAMA 271 (24):1953-1954. doi: 10.1001/jama.1994.03510480077038.

Martsolf, Grant, Kathryn R. Fingar, Rosanna Coffey, Ryan Kandrack, Tom Charland, Christine Eibner, Anne Elixhauser, Claudia Steiner, and Ateev Mehrotra. "Association Between the Opening of Retail Clinics and Low-Acuity Emergency Department Visits." Annals of Emergency Medicine 69 (4):397-403.e5. doi: 10.1016/j.annemergmed.2016.08.462.

Mehrotra, A., H. Liu, J. L. Adams, M. C. Wang, J. R. Lave, N. M. Thygeson, L. I. Solberg, and E. A. McGlynn. 2009. "Comparing costs and quality of care at retail clinics with that of other medical settings for 3 common illnesses." Ann Intern Med 151 (5):321-8.

Missouri Census Data Center. 2016. "All About ZIP Codes: 2010 Supplement." accessed July 1. http://mcdc.missouri.edu/geography/ZCTAs-2010.html.

Nadel, V. 1993. "Emergency departments: Unevenly affected by growth and change in patient use." US General Accounting Office: Report to the Chairman, Subcommittee on Health for Families and the Uninsured, Committee on Finance, US Senate. Washington, DC: US Government Printing Office.

Niska, Richard; Bhuiya, Farida; Xu, Jianmin 2010. "National Hospital Ambulatory Medical Care Survey: 2007 Emergency Department Summary." National Health Statistics Reports 26.

O'Malley, A. S. 2013. "After-hours access to primary care practices linked with lower emergency department use and less unmet medical need." Health Aff (Millwood) 32 (1):175-83. doi: 10.1377/hlthaff.2012.0494.

Pitts, S. R., J. M. Pines, M. T. Handrigan, and A. L. Kellermann. 2012. "National trends in emergency department occupancy, 2001 to 2008: effect of inpatient admissions versus emergency department practice intensity." Ann Emerg Med 60 (6):679-686.e3. doi: 10.1016/j.annemergmed.2012.05.014.

Rask, Kimberly J, Mark V Williams, Ruth M Parker, and Sally E McNagny. 1994. "Obstacles predicting lack of a regular provider and delays in seeking care for patients at an urban public hospital." JAMA 271 (24):1931-1933.

State of Arizona, ; . 2004. "Arizona Administrative Code Department of Health Services - Health Care Institutions: Licensing." http://apps.azsos.gov/public services/Title 09/9-10.pdf.

State of New Hampshire, ; . "New Hampshire Code of Administrative Rules." http://www.dhhs.nh.gov/oos/bhfa/documents/he-p806.pdf.

Steiner, Claudia, Anne Elixhauser, and Jenny Schnaier. 2001. "The healthcare cost and utilization project: an overview." Effective clinical practice: ECP 5 (3):143-151.

Stoimenoff, Laurel; , and Nate Newman. 2017. Urgent Care Industry White Paper 2018 (Unabridged): The Essential Role of the Urgent Care Center in Population Health.

Sussman, A., L. Dunham, K. Snower, M. Hu, O. S. Matlin, W. H. Shrank, N. K. Choudhry, and T. Brennan. 2013. "Retail clinic utilization associated with lower total cost of care." Am J Manag Care 19 (4):e148-57. 
United States Census Bureau. 2013. "2009-2013 American Community Survey 5-Year Estimates." accessed July 18. https://www.census.gov/programs-surveys/acs/.

Urgent Care Association of America. 2011. "The Case for Urgent Care." accessed July 14, 2015. https:/ucaoa.site-ym.com/resource/resmgr/Files/WhitePaperTheCaseforUrgentCa.pdf.

Urgent Care Association of America. 2015. "Industry Frequently Asked Questions." accessed June 01, 2016. http://www.ucaoa.org/general/custom.asp?page=IndustryFAQs.

Urgent Care Association of America. 2017. 2017 Benchmarking Report Summary: Headlines on Growth.

Urgent Care Association of America, ;. 2014. Urgent Care Association of America Releases 2014 Urgent Care Survey, Shows Major Industry-Wide Expansion.

Urgent Care Location, ;. 2013. Urgent Care Locations Provides Verified Urgent Care Directory.

Uscher-Pines, L., J. Pines, A. Kellermann, E. Gillen, and A. Mehrotra. 2013a. "Emergency department visits for nonurgent conditions: systematic literature review." Am J Manag Care 19 (1):47-59.

Uscher-Pines, Lori, Jesse Pines, Arthur Kellermann, Emily Gillen, and Ateev Mehrotra. 2013b. "Deciding to Visit the Emergency Department for Non-Urgent Conditions: A Systematic Review of the Literature." The American journal of managed care 19 (1):47.

Villaveces, Andrés, Ryan Mutter, Pamela L Owens, and Marguerite L Barrett. 2013. "Causes of injuries treated in the emergency department, 2010."

Weinick, R. M., R. M. Burns, and A. Mehrotra. 2010. "Many emergency department visits could be managed at urgent care centers and retail clinics." Health Aff (Millwood) 29 (9):1630-6. doi: 10.1377/hlthaff.2009.0748.

Welch, Shari J, James J Augustine, Li Dong, Lucy A Savitz, Gregory Snow, and Brent C James. 2012. "Volume-related differences in emergency department performance." The Joint Commission Journal on Quality and Patient Safety 38 (9):395-AP1.

Young, G. P., M. B. Wagner, A. L. Kellermann, J. Ellis, and D. Bouley. 1996. "Ambulatory visits to hospital emergency departments. Patterns and reasons for use. 24 Hours in the ED Study Group." JAMA 276 (6):460-5. 
Table 1. Summary Statistics for ZIP Codes with and without Urgent Care Centers

\begin{tabular}{lcc}
\hline & $\begin{array}{c}\text { Without any Urgent } \\
\text { Care Center }\end{array}$ & $\begin{array}{c}\text { With at Least One } \\
\text { Urgent Care Center }\end{array}$ \\
\hline Total Population (n) & 12,661 & $29,782^{* * *}$ \\
Age (median) & 42.0 & $40.7 * * *$ \\
Non-Hispanic White (\%) & 74.3 & $65.4^{* * *}$ \\
Household Income (median) & $\$ 28,997$ & $\$ 31,095^{* * *}$ \\
Did not earn high school degree (\%) & 12.7 & $11.5 * * *$ \\
Insurance Status (\%) & & 68.0 \\
$\quad$ Any Private & 67.5 & $14.5 * * *$ \\
$\quad$ Any Medicaid & 16.3 & $14.3 * *$ \\
\hline Uninsured & 13.3 & $835(14.9 \%)$ \\
\hline Observations (5,589) & $4,754(85.1 \%)$ & \\
\hline
\end{tabular}

Notes: Significance stars reflect results from means comparisons tests. ${ }^{*} \mathrm{p}<0.05,{ }^{* *} \mathrm{p}<0.01$, $* * * \mathrm{p}<0.001$ 
Figure 1. Unadjusted Hourly Rate of Non-emergent Emergency Department Visits in ZIP Codes with and without Urgent Care Centers

Panel A.

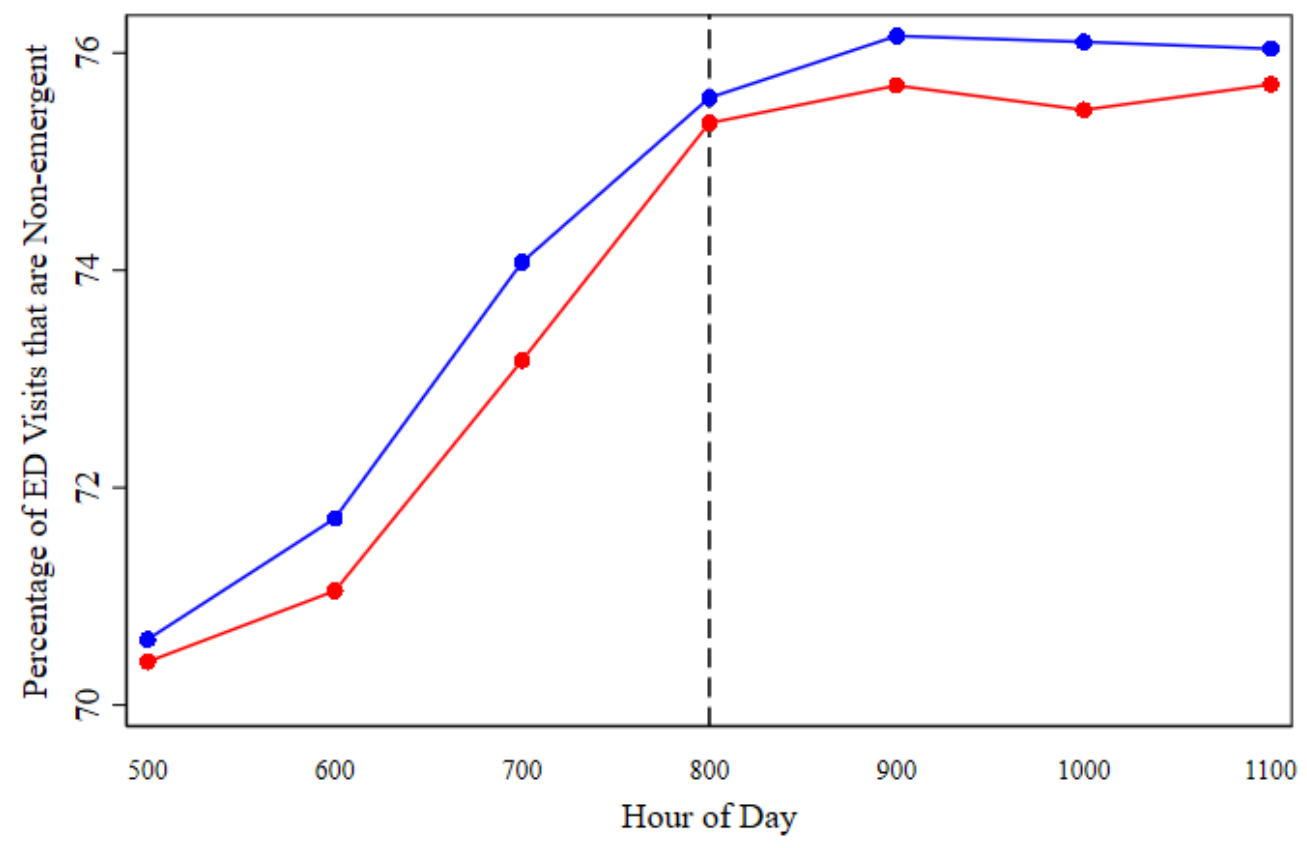

- With Urgent Care Centers - Without Urgent Care Centers

Panel B.

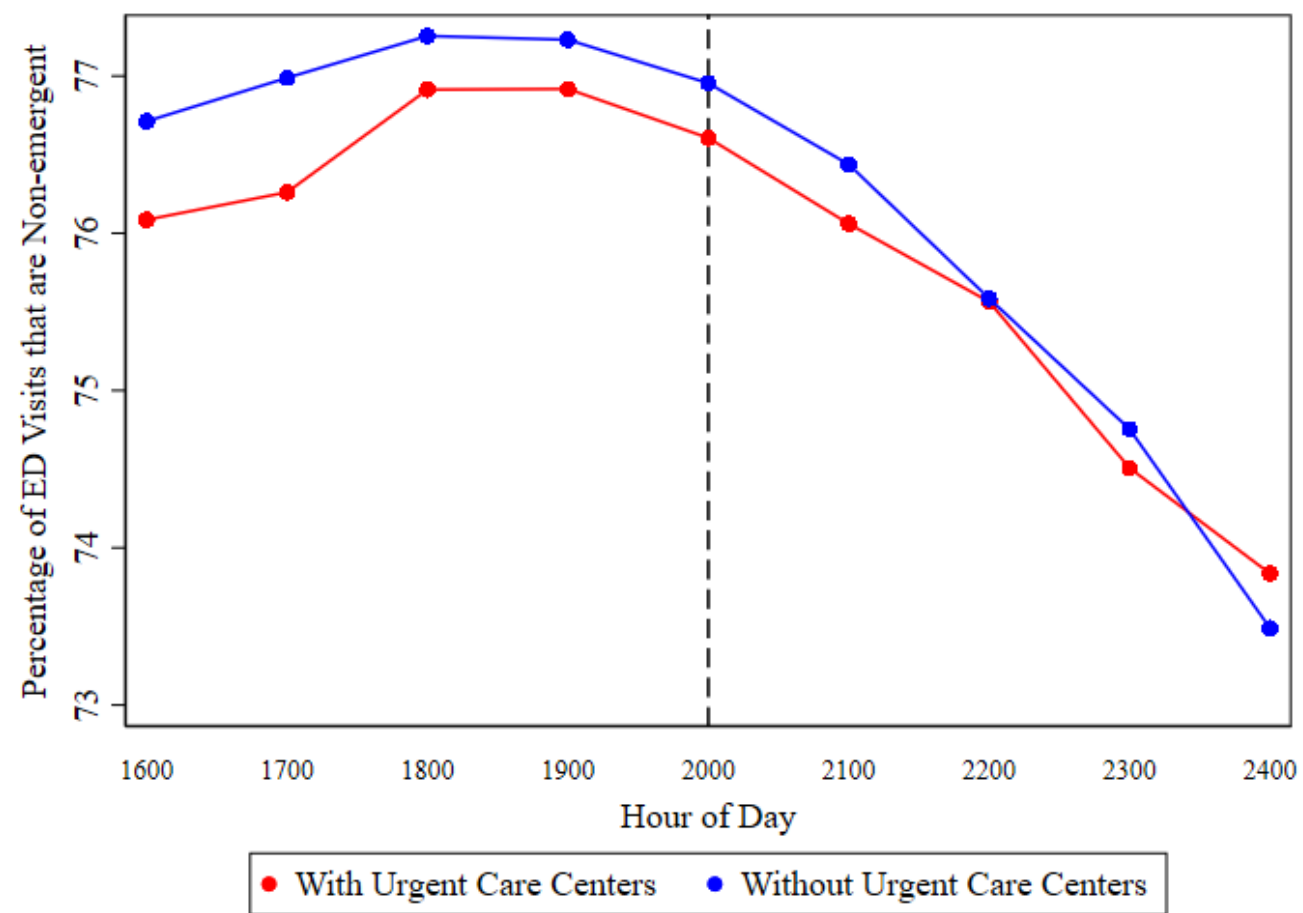


Table 2. Effect of Daily Urgent Care Center Opening/Closure on ZIP code Non-emergent Emergency Department Rates, by Urgent Care Center Status

\begin{tabular}{|c|c|c|c|c|}
\hline & \multicolumn{2}{|c|}{ Privately Insured } & \multicolumn{2}{|c|}{ Uninsured } \\
\hline & Coefficient & $\begin{array}{l}\% \text { Change } \\
\text { From } \\
\text { Baseline }\end{array}$ & Coefficient & $\begin{array}{l}\% \text { Change } \\
\text { From } \\
\text { Baseline }\end{array}$ \\
\hline \multicolumn{5}{|l|}{ Panel A. Daily Opening at 8 AM } \\
\hline Exactly 1 Urgent Care Center & $\begin{array}{l}-0.463 \\
(0.530)\end{array}$ & -0.68 & $\begin{array}{l}-0.003 \\
(0.632)\end{array}$ & 0.00 \\
\hline $\begin{array}{l}\text { Mean } \\
\text { Observations }\end{array}$ & $\begin{array}{l}68.02 \\
5,377\end{array}$ & & $\begin{array}{l}71.26 \\
4,486\end{array}$ & \\
\hline More than 1 Urgent Care Center & $\begin{array}{c}0.910 \\
(0.535)\end{array}$ & 1.34 & $\begin{array}{c}0.287 \\
(0.731)\end{array}$ & 0.40 \\
\hline $\begin{array}{l}\text { Mean } \\
\text { Observations }\end{array}$ & $\begin{array}{l}67.80 \\
5,189\end{array}$ & & $\begin{array}{l}71.22 \\
4,316\end{array}$ & \\
\hline \multicolumn{5}{|l|}{ Panel B. Daily Closure at 8 PM } \\
\hline Exactly 1 Urgent Care Center & $\begin{array}{c}0.575 \\
(0.366)\end{array}$ & 0.81 & $\begin{array}{c}0.288 \\
(0.504)\end{array}$ & 0.40 \\
\hline $\begin{array}{l}\text { Mean } \\
\text { Observations }\end{array}$ & $\begin{array}{l}70.57 \\
5,763\end{array}$ & & $\begin{array}{l}72.82 \\
5,007\end{array}$ & \\
\hline More than 1 Urgent Care Center & $\begin{array}{l}1.01 * * \\
(0.452)\end{array}$ & 1.43 & $\begin{array}{c}0.179 \\
(0.497)\end{array}$ & 0.25 \\
\hline $\begin{array}{l}\text { Mean } \\
\text { Observations }\end{array}$ & $\begin{array}{l}70.58 \\
5,527\end{array}$ & & $\begin{array}{l}72.82 \\
5,290\end{array}$ & \\
\hline \multicolumn{5}{|c|}{$\begin{array}{l}\text { Notes: The analytic time window is the hour immediately prior to opening/closure, and the hour } \\
\text { immediately after. Means reflect the adjusted percentage of ED visits in the ZIP code that are } \\
\text { classified as not truly emergent. Standard errors are in parentheses. All standard errors are } \\
\text { clustered at the Hospital Service Area level. }{ }^{*} \mathrm{p}<0.05,{ }^{*} \mathrm{p}<0.01, * * * \mathrm{p}<0.001\end{array}$} \\
\hline
\end{tabular}


Table 3. Effect of Daily Urgent Care Center Closure on ZIP Code Non-emergent Emergency Department Rates, by Varying Percentages of Non-emergent Injuries

\begin{tabular}{|c|c|c|c|c|}
\hline & \multicolumn{2}{|c|}{ Privately Insured } & \multicolumn{2}{|c|}{ Uninsured } \\
\hline $\begin{array}{l}\text { Percent of Injury Visits } \\
\text { Assumed to be Non- } \\
\text { Emergent }\end{array}$ & Coefficient & $\begin{array}{c}\text { \% Change From } \\
\text { Baseline }\end{array}$ & Coefficient & $\begin{array}{c}\text { \% Change From } \\
\text { Baseline }\end{array}$ \\
\hline $25 \%$ & $\begin{array}{c}0.824 \\
(0.569)\end{array}$ & 1.5 & $\begin{array}{c}0.449 \\
(0.677)\end{array}$ & 0.74 \\
\hline Mean & 54.82 & & 60.37 & \\
\hline Observations & 6,048 & & 5,290 & \\
\hline $50 \%$ & $\begin{array}{c}0.732 \\
(0.572)\end{array}$ & 1.15 & $\begin{array}{l}-0.0846 \\
(0.616)\end{array}$ & -0.13 \\
\hline Mean & 63.44 & & 67.05 & \\
\hline Observations & 6,048 & & 5,290 & \\
\hline $75 \%$ & $\begin{array}{l}1.237^{*} \\
(0.480)\end{array}$ & 1.72 & $\begin{array}{l}-0.0460 \\
(0.570)\end{array}$ & -0.06 \\
\hline Mean & 72.11 & & 73.48 & \\
\hline Observations & 6,048 & & 5,290 & \\
\hline $100 \%$ & $\begin{array}{l}\mathbf{0 . 9 7 8}^{* * *} \\
(0.349)\end{array}$ & 1.21 & $\begin{array}{l}0.0810 \\
(0.450)\end{array}$ & 0.1 \\
\hline Mean & $80.83 \mathrm{~s}$ & & 79.85 & \\
\hline Observations & 6,048 & & 5,290 & \\
\hline
\end{tabular}


Appendix: Weekday Opening and Closure Times of Urgent Care Centers, 6 states
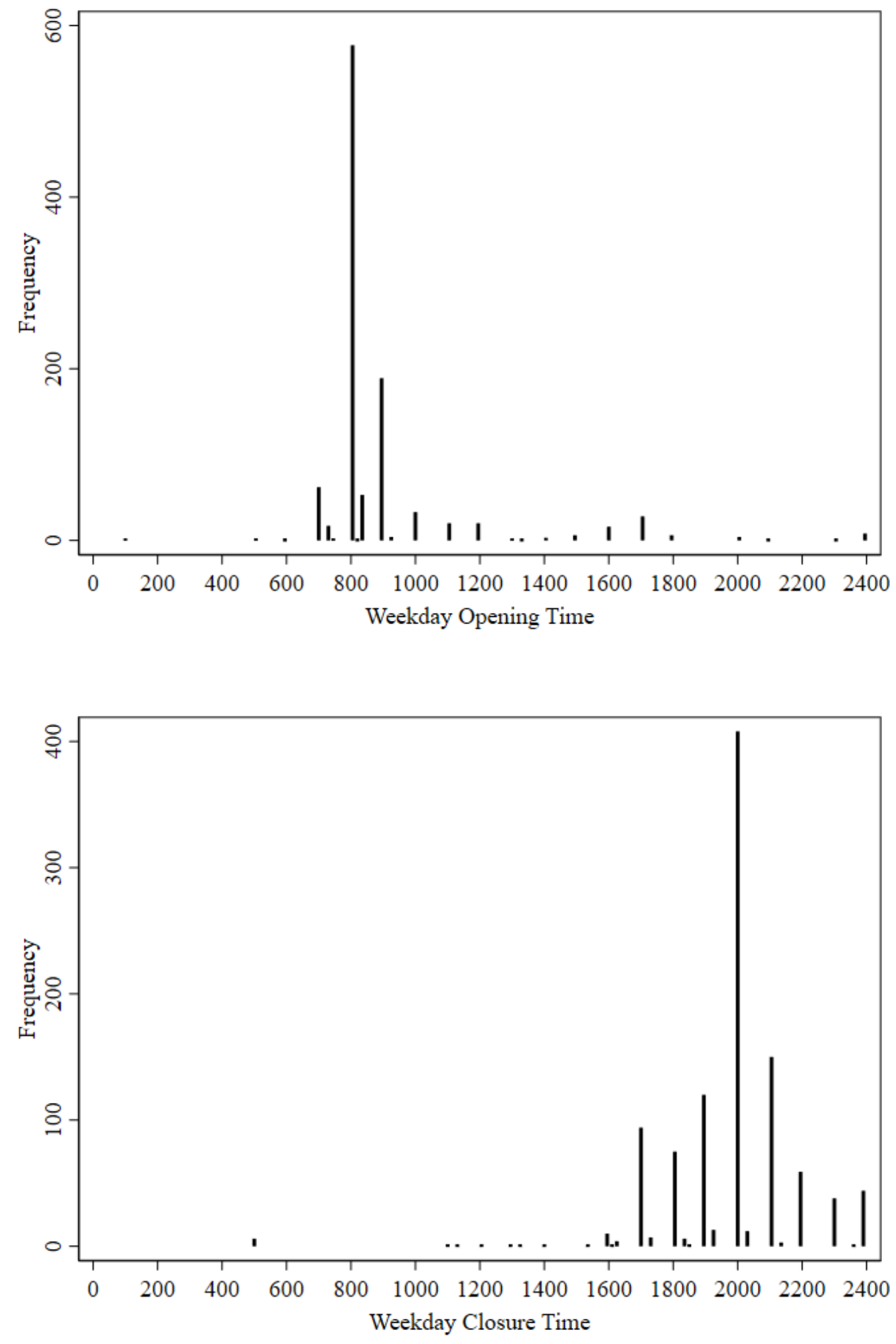